\title{
A delayed Holling type III functional response predator-prey system with impulsive perturbation on the prey
}

"Correspondence:

dylww1958@163.com School of Mathematics and

Statistics, Qiannan Normal College for Nationalities, Duyun, Guizhou 558000, China

\section{Springer}

\begin{abstract}
A Holling type III functional response predator-prey system with constant gestation time delay and impulsive perturbation on the prey is investigated. The sufficient conditions for the global attractivity of a predator-extinction periodic solution are obtained by the theory of impulsive differential equations, i.e. the impulsive period is less than the critical value $T_{1}^{*}$. The conditions for the permanence of the system are investigated, i.e. the impulsive period is larger than the critical value $T_{2}^{*}$. Numerical examples show that the system has very complex dynamic behaviors, including (1) high-order periodic and quasi-periodic oscillations, (2) period-doubling and -halving bifurcations, and (3) chaos and attractor crises. Further, the importance of the impulsive period, the gestation time delay, and the impulsive perturbation proportionality constant are discussed. Finally, the impulsive control strategy and the biological implications of the results are discussed.
\end{abstract}

Keywords: predator-prey system; impulsive perturbation; time delay; extinction; permanence; chaos

\section{Introduction}

The time delay population dynamics system describes the current state of the population not only related to the current state but also related to the state of the population in the past. That is to say, the time delay effect is very important in population dynamics, which tends to destabilize the positive equilibria and cause a loss of stability, bifurcate into various periodic solutions, even make chaotic oscillations. Recently, there has been much work dealing with time delayed population systems (see [1-12]). For example, a stagestructured prey-predator system with time delay and Holling type-III functional response is considered by Wang et al. [4], the existence and properties of the Hopf bifurcations are established. A delayed eco-epidemiology model with Holling-III functional response was instigated by Zou et al. [5], and one found that time delay may lead to Hopf bifurcation under certain conditions. The Hopf bifurcation of a delayed predator-prey system with Holling type-III functional response also has been considered in [6-8]. The existence of positive periodic solutions of a delayed nonautonomous prey-predator system with Holling type-III functional response was considered in [9-11], by using the continuation theorem of coincidence degree theory. Therefore, time delays would make the prey-

(c) $2016 \mathrm{Li}$ and Liu. This article is distributed under the terms of the Creative Commons Attribution 4.0 International License (http://creativecommons.org/licenses/by/4.0/), which permits unrestricted use, distribution, and reproduction in any medium, provided you give appropriate credit to the original author(s) and the source, provide a link to the Creative Commons license, and indicate if changes were made. 
predator system subject to periodic oscillations via a local Hopf bifurcation, and destroy the stability of the system.

Recently, more and more authors have discussed the impulsive perturbation on preypredator systems, since the system would be stabilized by impulsive effects, and would make the system subject to complex dynamical behaviors [13-21]. For example, a predatorprey model with impulsive effect and generalized Holling type III functional response was studied by Su et al. [15], and the sufficient conditions for the existence of a pest-eradication periodic solution and permanence of the system are obtained. The existence of positive periodic solutions of the nonautonomous prey-predator system with Holling type III functional response and impulsive perturbation is considered in $[17,18]$. By using a continuation theorem of coincidence degree theory, the sufficient conditions for the existence of a positive periodic solution are obtained for the system.

Note that harmful delays would destroy the stability of the system via bifurcations and even lead the system to extinction. At this point, the impulsive control strategies can be considered, which can both improve the stability of the system and control the amplitude of the bifurcated periodic solution effectively. For example, a time delayed Holling type II functional response prey-predator system with impulsive perturbations is investigated by Jia et al. [22], and the problems of the predator-extinction periodic solution and the permanence of the system are investigated. So, how does the dynamical behavior go when the delayed system with impulsive effect? Especially, what would happen for the delayed predator-prey system with Holling type III functional response under impulsive perturbation?

Motivated by the aforementioned observations, we assume the predator needs a certain time to gestate the prey, and we consider the following delayed Holling type III functional response prey-predator system with impulsive perturbation on the prey:

$$
\left\{\begin{array}{r}
x^{\prime}(t)=r x(t)\left(1-\frac{x(t)}{K}\right)-\frac{\alpha x^{2}(t) y(t)}{1+\beta x^{2}(t)}, \\
y^{\prime}(t)=\frac{k \alpha x^{2}(t-\tau) y(t-\tau)}{1+\beta x^{2}(t-\tau)}-d y(t),
\end{array}\right\} \quad t \neq n T,
$$

where $x(t)$ and $y(t)$ are the prey and predator populations at time $t$, respectively; $r, K, \alpha$, $\beta, k, d$ are positive. $r$ is the intrinsic rate of increase of the prey, $K$ is the carrying capacity of the prey. $\alpha$ is the predation coefficient of the predator, which reflects the size of the predator's ability, and $\beta$ is predation regulation factor (saturation factor) of the predator. $d$ is the death rate of the predator, $k(0<k<1)$ is the rate of conversing prey into predator. $\Delta x(t)=x\left(t^{+}\right)-x(t), \Delta y(t)=y\left(t^{+}\right)-y(t), T$ is the impulsive periodic. $n \in \mathbb{N}_{+}, \mathbb{N}_{+}=\{1,2, \ldots\}$, $p>0$ is the proportionality constant which represents the rate of mortality due to the applied pesticide. The initial conditions for system (1) are

$$
\left(\phi_{1}(s), \phi_{2}(s)\right) \in C_{+}=C\left([-\tau, 0], \mathbb{R}_{+}^{2}\right), \quad \phi_{i}(0)>0(i=1,2) .
$$

The paper is arranged as follows. Some notations and lemmas are given in the next section, and we consider the existence and global attraction of the predator-extinction periodic solutions of the system. The sufficient conditions for the permanence of the system are given by using the theory on impulsive and delay differential equation. Numerical 
examples are given to support the theoretical research, and some complex dynamic behaviors are shown. For example, we see period-halving and period-doubling bifurcations, periodic and high-order quasi-periodic oscillations, even chaotic oscillation. The importance of the impulsive period $T$, the gestation time delay $\tau$, and the impulsive perturbation proportionality constant $p$ are discussed. Finally, the impulsive control strategy and biological implications of the results are discussed.

\section{Preliminaries}

Let $\mathbb{R}_{+}=[0, \infty), \mathbb{R}_{+}^{2}=\left\{x \in \mathbb{R}^{2} \mid x \geq 0\right\}$. Denote by $f=\left(f_{1}, f_{2}\right)$ the map defined by the right hand of the first two equations of system (1), and let $\mathbb{N}$ be the set of all non-negative integers. Let $V: \mathbb{R}_{+} \times \mathbb{R}_{+}^{2} \rightarrow \mathbb{R}_{+}$, then $V$ is said to belong to class $V_{0}$ if:

(1) $V$ is continuous in $(t, x) \in(n T,(n+1) T] \times \mathbb{R}_{+}^{2}$ and for each $x \in \mathbb{R}_{+}^{2}, n \in \mathbb{N}$, $\lim _{(t, y) \rightarrow\left(n T^{+}, x\right)} V(t, y)=V\left(n T^{+}, x\right)$ exists.

(2) $V$ is locally Lipschitzian in $x$.

Definition 2.1 Let $V \in V_{0}$, then for $(t, x) \in(n T,(n+1) T] \times \mathbb{R}_{+}^{2}$, the upper right derivative of $V(t, x)$ with respect to the impulsive differential system (1) is defined as

$$
D^{+} V(t, x)=\lim _{h \rightarrow 0^{+}} \sup \frac{1}{h}[V(t+h, x+h f(t, x))-V(t, x)]
$$

Definition 2.2 System (1) is said to be permanent if there exist two positive constants $m, M$, and $T_{0}$ such that each positive solution $X(t)=(x(t), y(t))$ of the system (1) satisfies $m \leq x(t) \leq M, m \leq y(t) \leq M$ for all $t>T_{0}$.

The solution of system (1) is a piecewise continuous function $x: \mathbb{R}_{+} \mapsto \mathbb{R}_{+}^{2}, x(t)$ is continuous on $(n T,(n+1) T], n \in \mathbb{N}$, and $x\left(n T^{+}\right)=\lim _{t \rightarrow n T^{+}} x(t)$ exists, the smoothness properties of $f$ guarantee the global existence and uniqueness of solutions of system (1), for details see $[23,24]$.

Lemma 2.1 Let $X(t)$ be a solution of system (1) with $X\left(0^{+}\right) \geq 0$, then $X(t) \geq 0$ for all $t \geq 0$ and further $X(t)>0$ for all $t \geq 0$ if $X\left(0^{+}\right)>0$.

Lemma $2.2[23]$ Suppose $V \in V_{0}$. Assume that

$$
\begin{cases}D^{+} V(t, x) \leq g(t, V(t, x)), & t \neq n T \\ V\left(t, x\left(t^{+}\right)\right) \leq \psi_{n}(V(t, x)), & t=n T\end{cases}
$$

where $g: \mathbb{R}_{+} \times \mathbb{R}_{+} \mapsto \mathbb{R}$ is continuous in $(n T,(n+1) T] \times \mathbb{R}_{+}$and for $u \in \mathbb{R}_{+}, n \in \mathbb{N}$, $\lim _{(t, y) \rightarrow\left(n T^{+}, u\right)}=g\left(n T^{+}, u\right)$ exists, $\psi_{n}: \mathbb{R}_{+} \rightarrow \mathbb{R}_{+}$is non-decreasing. Let $r(t)$ be the maximal solution of the scalar impulsive differential equation

$$
\begin{cases}u^{\prime}(t)=g(t, u(t)), & t \neq n T \\ u\left(t^{+}\right)=\psi_{n}(u(t)), & t=n T, \\ u\left(0^{+}\right)=u_{0}, & \end{cases}
$$

existing on $[0, \infty)$. Then $V\left(0^{+}, x_{0}\right) \leq u_{0}$ implies that $V(t, x(t)) \leq r(t), t \geq 0$ where $X(t)$ is any solution of system (1). 
We consider the following subsystem of system (1):

$$
\begin{cases}x^{\prime}(t)=r x(t)\left(1-\frac{x(t)}{K}\right), & t \neq n T, \\ \Delta x(t)=-p x(t), & t=n T .\end{cases}
$$

Clearly, if $(1-p) e^{r T}>1$,

$$
x^{*}(t)=\frac{x_{0}^{*}}{\left(1-\frac{x_{0}^{*}}{K}\right) e^{-r(t-n T)}+\frac{x_{0}^{*}}{K}}, \quad t \in(n T,(n+1) T], \quad x_{0}^{*}=\frac{K\left[(1-p) e^{r T}-1\right]}{e^{r T}-1},
$$

is a globally asymptotically stable positive periodic solution of system (5) [22].

Lemma 2.3 If $(1-p) e^{r T}>1$, system (1) has a predator-extinction periodic solution $X(t)=$ $\left(x^{*}(t), 0\right)$ for $t \in(n T,(n+1) T]$, and for any solution $X(t)=(x(t), y(t))$ of system $(1)$, we have $x(t) \rightarrow x^{*}(t)$ as $t \rightarrow+\infty$.

Lemma 2.4 Consider the following delay differential equation [25]:

$$
x^{\prime}(t)=a x(t-\tau)-b x(t)
$$

where $a, b, \tau$ are all positive constants and $x(t)>0$ for $t \in[-\tau, 0]$.

(i) If $a<b$, then $\lim _{t \rightarrow+\infty} x(t)=0$.

(ii) If $a>b$, then $\lim _{t \rightarrow+\infty} x(t)=+\infty$.

\section{Extinction and permanence}

Denote

$$
X_{1}^{*}=\frac{K\left[(1-p) e^{r T}-1\right]}{(1-p)\left(e^{r T}-1\right)}, \quad \Re_{1}=\frac{k \alpha X_{1}^{* 2}}{d\left(1+\beta X_{1}^{* 2}\right)}
$$

Theorem 3.1 If $\Re_{1}<1$ and $(1-p) e^{r T}>1$, then the predator-extinction periodic solution $X(t)=\left(x^{*}(t), 0\right)$ of system $(1)$ is globally attractive.

Proof Let $X(t)=(x(t), y(t))$ be any solution of system (1) with initial conditions (2). By the second equation of system (1), we get

$$
y^{\prime}(t) \leq \frac{k \alpha}{\beta} y(t-\tau)-d y(t)
$$

Consider the following delayed comparison equation:

$$
z^{\prime}(t)=\frac{k \alpha}{\beta} z(t-\tau)-d z(t)
$$

If $k \alpha<d \beta$, then $\Re_{1}<1$. According to Lemma 2.4, we have $\lim _{t \rightarrow+\infty} z(t)=0$. Note that $y(t)=z(t)=\phi_{2}(t)>0$ for all $t \in[-\tau, 0]$, then we have $y(t) \rightarrow 0$ as $t \rightarrow+\infty$. We assume that $k \alpha>d \beta$ in the rest of this paper. $\Re_{1}<1$ and we note that $\frac{k \alpha z^{2}}{1+\beta z^{2}}$ is a monotonically 
increasing function about variable $z$. Therefore, there exists a sufficiently small positive constant $\varepsilon$ such that

$$
\frac{k \alpha\left(X_{1}^{*}+\varepsilon\right)^{2}}{1+\beta\left(X_{1}^{*}+\varepsilon\right)^{2}}-d<0
$$

By the first and third equations of system (1), we have

$$
\begin{cases}x^{\prime}(t) \leq r x(t)\left(1-\frac{x(t)}{K}\right), & t \neq n T \\ \Delta x(t)=-p x(t), & t=n T\end{cases}
$$

then we consider the following comparison system:

$$
\begin{cases}z^{\prime}(t)=r z(t)\left(1-\frac{z(t)}{K}\right), & t \neq n T \\ \Delta z(t)=-p z(t), & t=n T\end{cases}
$$

with $z\left(0^{+}\right)=z(0)=x(0)$. Recalling system (5), we obtain a unique globally asymptotically stable positive $T$-periodic solution of system (6), where

$$
z^{*}(t)=\frac{K}{\left[\frac{e^{r T}-1}{(1-p) e^{r T}-1}-1\right] e^{-r(t-n T)}+1}, \quad t \in(n T,(n+1) T] .
$$

Then, there exists an arbitrarily small positive constant $\varepsilon$ and $n_{1} \in \mathbb{N}$ such that

$$
x(t) \leq z^{*}(t)+\varepsilon \leq \frac{K}{\left[\frac{e^{r T}-1}{(1-p) e^{r T}-1}-1\right] e^{-r T}+1}+\varepsilon=X_{1}^{*}+\varepsilon \triangleq \eta
$$

for all $t \geq n_{1} T$. From (7) and the second equation of system (1), we have

$$
y^{\prime}(t) \leq \frac{k \alpha \eta^{2}}{1+\beta \eta^{2}} y(t-\tau)-d y(t)
$$

for $t \geq n_{1} T+\tau$. Consider the following delayed comparison equation:

$$
z^{\prime}(t)=\frac{k \alpha \eta^{2}}{1+\beta \eta^{2}} z(t-\tau)-d z(t)
$$

According to Lemma 2.4, we get $\lim _{t \rightarrow+\infty} z(t)=0$. Note that $y(t)=z(t)=\phi_{2}(t)>0$ for all $t \in[-\tau, 0]$, then we have $y(t) \rightarrow 0$ as $t \rightarrow+\infty$.

Without loss of generality, we may suppose that $0<y(t)<\varepsilon$ and $r_{1}=r-\alpha K \varepsilon>0, K_{1}=$ $r_{1} K / r$ for $\varepsilon$ small enough and $t \geq 0$. By the first equation of system (1), we obtain

$$
x^{\prime}(t) \geq r_{1} x(t)\left(1-\frac{x(t)}{K_{1}}\right) .
$$

Then we have $z_{1}^{*}(t) \rightarrow x^{*}(t)$ as $\varepsilon \rightarrow 0(t \rightarrow+\infty)$, where $z_{1}^{*}(t)$ is the unique periodic solution of the following comparison system:

$$
\begin{cases}z_{1}^{\prime}(t)=r_{1} z_{1}(t)\left(1-\frac{z_{1}(t)}{K_{1}}\right), & t \neq n T, \\ \Delta z_{1}(t)=-p z_{1}(t), & t=n T,\end{cases}
$$


with initial condition $z_{1}\left(0^{+}\right)=x\left(0^{+}\right)$. From (8), we get

$$
z_{1}^{*}(t)=\frac{z_{1}^{*}}{\left(1-\frac{z_{1}^{*}}{K_{1}}\right) e^{-r_{1}(t-n T)}+\frac{z_{1}^{*}}{K_{1}}}, \quad t \in(n T,(n+1) T],
$$

where

$$
z_{1}^{*}=\frac{K_{1}\left[(1-p) e^{r_{1} T}-1\right]}{e^{r_{1} T}-1} .
$$

Therefore, for any $\varepsilon_{1}>0$ there exists a $T_{0}>0$ such that

$$
x(t)>z_{1}^{*}(t)-\varepsilon_{1}
$$

for $t>T_{0}$.

By the first equation of system (1), we get

$$
x(t) \leq r x(t)\left(1-\frac{x(t)}{K}\right) .
$$

Consider the following comparison system:

$$
\begin{cases}z_{2}^{\prime}(t)=r z_{2}(t)\left(1-\frac{z_{2}(t)}{K}\right), & t \neq n T \\ \Delta z_{2}(t)=-p z_{2}(t), & t=n T\end{cases}
$$

with initial condition $z_{2}\left(0^{+}\right)=x\left(0^{+}\right)$. Then, we have

$$
x(t)<z_{2}^{*}(t)+\varepsilon_{1}
$$

as $t \rightarrow+\infty$ and $z_{2}^{*}(t)=x^{*}(t)$, where $z_{2}^{*}(t)$ is the unique positive periodic solution of (10). Let $\varepsilon \rightarrow 0$, and by (9) and (11), we have

$$
x^{*}(t)-\varepsilon_{1}<x(t)<x^{*}(t)+\varepsilon_{1}
$$

for sufficiently large $t$. This implies $x(t) \rightarrow x^{*}(t)$ as $t \rightarrow+\infty$.

Theorem 3.2 There exists a constant $Y_{0}=M_{1} / d-k K>0$, such that $x(t) \leq K$ and $y(t) \leq Y_{0}$ for any solution $X(t)=(x(t), y(t))$ of system (1) with all t large enough.

Proof Let $V(t)=k x(t)+y(t+\tau)$. Note that $V \in V_{0}$. We get $V^{\prime}(t)$ by calculating the upper right derivative of $V(t)$ along a solution of system (1):

$$
V^{\prime}(t)=k r x(t)\left(1-\frac{x(t)}{K}\right)-d y(t+\tau)=-d V(t)+k x(t)\left[d+r\left(1-\frac{x(t)}{K}\right)\right] .
$$

Let $M_{1}=\max \left\{K k d, \frac{K(r+d)^{2}}{4}\right\}$, then we have

$$
\frac{\mathrm{d} V(t)}{\mathrm{d} t} \leq-d V(t)+M_{1}
$$


Consider the following differential inequalities:

$$
\begin{cases}V^{\prime}(t) \leq-d V(t)+M_{1}, & t \neq n T, \\ V\left(t^{+}\right) \leq V(t), & t=n T,\end{cases}
$$

according to Lemma 2.2, we have

$$
V(t) \leq\left(V\left(0^{+}\right)-\frac{M_{1}}{d}\right) e^{-d t}+\frac{M_{1}}{d}
$$

Therefore $\lim _{t \rightarrow+\infty} V(t) \leq M_{1} / d \triangleq M$, then $V(t)$ is ultimately bounded. Therefore, any positive solution of system (1) is uniformly ultimately bounded.

\section{Denote}

$$
\Re_{2}=\frac{k \alpha x_{0}^{* 2}}{d\left(1+\beta x_{0}^{* 2}\right)}
$$

Theorem 3.3 If $\Re_{2}>1$ and $r>\alpha K Y_{0}$, then system (1) is uniformly persistent.

Proof Suppose that $X(t)=(x(t), y(t))$ is each positive solution of system (1) with initial conditions (2). Rewrite the second equation of system (1) as follows:

$$
\frac{\mathrm{d} y(t)}{\mathrm{d} t}=\left(\frac{k \alpha x^{2}(t)}{1+\beta x^{2}(t)}-d\right) y(t)-k \alpha \frac{\mathrm{d}}{\mathrm{d} t} \int_{t-\tau}^{t} \frac{x^{2}(\theta)}{1+\beta x^{2}(\theta)} y(\theta) \mathrm{d} \theta
$$

Define

$$
V(t)=y(t)+k \alpha \int_{t-\tau}^{t} \frac{x^{2}(\theta)}{1+\beta x^{2}(\theta)} y(\theta) \mathrm{d} \theta .
$$

Calculating $V^{\prime}(t)$ along the solution of system (1), we have

$$
V^{\prime}(t)=d\left(\frac{k \alpha}{d} \frac{x^{2}(t)}{1+\beta x^{2}(t)}-1\right) y(t)
$$

Since $\Re_{2}>1$, there exist two positive constants $m_{2}^{*}$ and $\varepsilon_{1}$ small enough such that

$$
\frac{k \alpha}{d} \frac{\rho^{2}}{1+\beta \rho^{2}}>1
$$

where

$$
\begin{array}{ll}
\rho=\frac{K_{2}\left[(1-p) e^{r_{2} T}-1\right]}{e^{r_{2} T}-1}-\varepsilon_{1}>0, & r_{2}=r-\alpha K m_{2}^{*}>0, \\
K_{2}=\frac{K r_{2}}{r}>0, & 0<m_{2}^{*}<\frac{r T+\ln (1-p)}{\alpha K T} .
\end{array}
$$

We claim that: for any positive constant $t_{0}$, the inequality $y(t)<m_{2}^{*}$ cannot hold for all $t \geq t_{0}$. Otherwise, we can choose a positive constant $t_{0}$ such that $y(t)<m_{2}^{*}$ for all $t \geq t_{0}$. 
By the first and third equations of system (1), we obtain

$$
\begin{cases}x^{\prime}(t) \geq r_{2} x(t)\left(1-\frac{x(t)}{K_{2}}\right), & t \neq n T, \\ \Delta x(t)=-p x(t), & t=n T .\end{cases}
$$

Then, we have $z_{2}^{*}(t) \leq x(t)$, where $z_{2}^{*}(t)$ is an unique positive solution of the following comparison system:

$$
\begin{cases}z_{2}^{\prime}(t)=r_{2} z_{2}(t)\left(1-\frac{z_{2}(t)}{K_{2}}\right), & t \neq n T, \\ \Delta z_{2}(t)=-p z_{2}(t), & t=n T\end{cases}
$$

with initial condition $z_{2}\left(0^{+}\right)=x\left(0^{+}\right)$. From (15), we obtain

$$
z_{2}^{*}(t)=\frac{z_{2}^{*}}{\left(1-\frac{z_{2}^{*}}{K_{2}}\right) e^{-r_{2}(t-n T)}+\frac{z_{2}^{*}}{K_{2}}},
$$

for $t \in(n T,(n+1) T]$, where

$$
z_{2}^{*}=\frac{K_{2}\left[(1-p) e^{r_{2} T}-1\right]}{e^{r_{2} T}-1} .
$$

Then, for any $\varepsilon_{1}>0$ there exists a $T_{1}>0$ such that

$$
x(t)>z_{2}^{*}(t)-\varepsilon_{1} \geq z_{2}^{*}-\varepsilon_{1} \triangleq \rho,
$$

for $t>T_{1}$.

When $t \geq T_{1}$, from (12) and (16) we get

$$
V^{\prime}(t)>d\left(\frac{k \alpha}{d} \frac{\rho^{2}}{1+\beta \rho^{2}}-1\right) y(t) .
$$

Let $y_{1}=\min \left\{y(t) \mid T_{1} \leq t \leq T_{1}+\tau\right\}$. We show that $y(t) \geq y_{1}$ for all $t \geq T_{1}$. Otherwise, there exists a nonnegative constant $T_{2}$ such that

$$
y(t) \geq y_{1} \quad\left(T_{1} \leq t \leq T_{1}+T_{2}+\tau\right), \quad y\left(T_{1}+T_{2}+\tau\right)=y_{1}, \quad y^{\prime}\left(T_{1}+T_{2}+\tau\right) \leq 0 .
$$

Thus, by the second equation of system (1), (13), and (17), we obtain

$$
y^{\prime}\left(T_{1}+T_{2}+\tau\right)>d\left(\frac{k \alpha}{d} \frac{\rho^{2}}{1+\beta \rho^{2}}-1\right) y_{1}>0
$$

which is a contradiction. Hence, we get $y(t)>y_{1}>0$ for all $t \geq T_{1}$. From (17), we have

$$
V^{\prime}(t)>d\left(\frac{k \alpha}{d} \frac{\rho^{2}}{1+\beta \rho^{2}}-1\right) y(t)>0,
$$

which implies $V(t) \rightarrow+\infty$ as $t \rightarrow+\infty$. This is a contradiction to $V(t) \leq M$. Then, for any given positive constant $t_{0}$, the inequality $y(t)<m_{2}^{*}$ cannot hold for all $t \geq t_{0}$. 


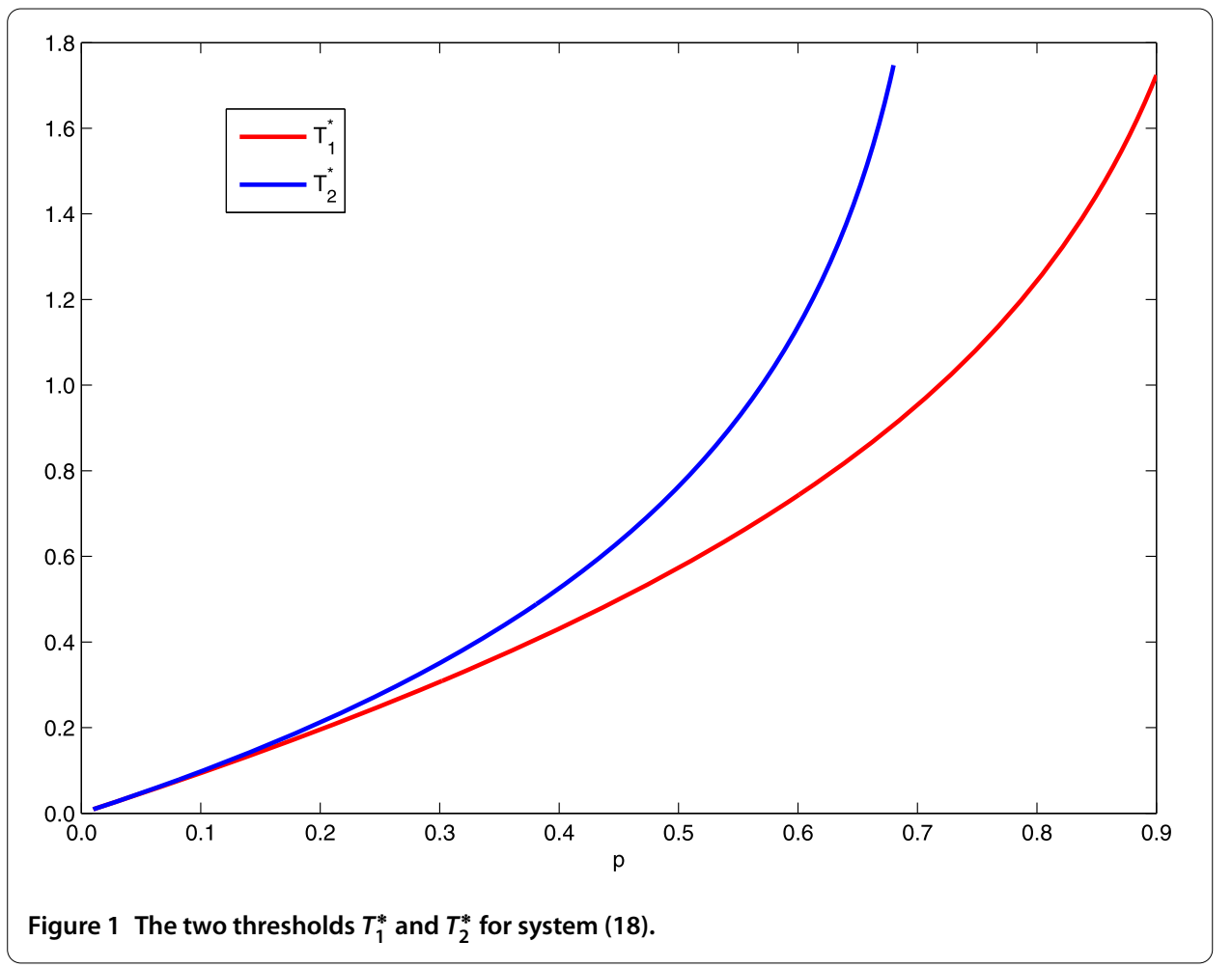

On the one hand, if $y(t) \geq m_{2}^{*}$ holds true for all sufficiently large $t$, then our aim is reached. On the other hand, suppose $y(t)$ is oscillatory about $m_{2}^{*}$. Let $m_{2}=\min \left\{m_{2}^{*} / 2\right.$, $\left.m_{2}^{*} \exp (-d \tau)\right\}$ and we will prove that $y(t) \geq m_{2}$. There exist two positive constants $\bar{t}$ and $\omega$ such that $y(\bar{t})=y(\bar{t}+\omega)=m_{2}^{*}$ and $y(t)<m_{2}^{*}$ for $t \in(\bar{t}, \bar{t}+\omega)$. The inequality $x(t)>\rho$ holds true for $t \in(\bar{t}, \bar{t}+\omega)$ when $\bar{t}$ is large enough.

Since there is no impulsive effect on $y(t), y(t)$ is uniformly continuous. Then, there exists a constant $T_{3}$ (with $0<T_{3}<\tau$ and $T_{3}$ is dependent of the choice of $\bar{t}$ ) such that $y(t)>m_{2}^{*} / 2$ for all $t \in\left[\bar{t}, \bar{t}+T_{3}\right]$.

If $\omega \leq T_{3}$, our aim is reached. If $T_{3}<\omega \leq \tau$, by the second equation of system (1) we get $y^{\prime}(t) \geq-d y(t)$ for $t \in(\bar{t}, \bar{t}+\omega]$. Then we get $y(t) \geq m_{2}^{*} \exp (-d \tau)$ for $\bar{t}<t \leq \bar{t}+\omega \leq \bar{t}+\tau$ since $y(t)=m_{2}^{*}$. Therefore, $y(t) \geq m_{2}$ for $t \in(\bar{t}, \bar{t}+\omega]$.

If $\omega \geq \tau$, from the second equation of system (1), then we get $y(t) \geq m_{2}$ for $t \in(\bar{t}, \bar{t}+\tau]$. Thus, we have $y(t) \geq m_{2}$ for $t \in[\bar{t}+\tau, \bar{t}+\omega]$. According to the above proof. Since the interval $[\bar{t}, \bar{t}+\omega]$ is arbitrarily chosen, we have $y(t) \geq m_{2}$ for sufficiently large $t$. From our arguments above, the choice of $m_{2}$ is independent of the positive solution of system (1) which shows that $y(t) \geq m_{2}$ holds for sufficiently large $t$.

By Theorem 3.2, we obtain $y(t) \leq Y_{0}$ for sufficiently large $t$. Therefore, by the first equation of system (1), we get

$$
x^{\prime}(t) \geq r_{3} x(t)\left(1-\frac{x(t)}{K_{3}}\right)
$$

for sufficiently large $t$, where $r_{3}=r-\alpha K Y_{0}$ and $K_{3}=K r_{3} / r$. Therefore, we have $x(t) \geq$ $z_{3}^{*}(t)$, where $z_{3}^{*}(t)$ is a unique globally asymptotically stable positive periodic solution of 


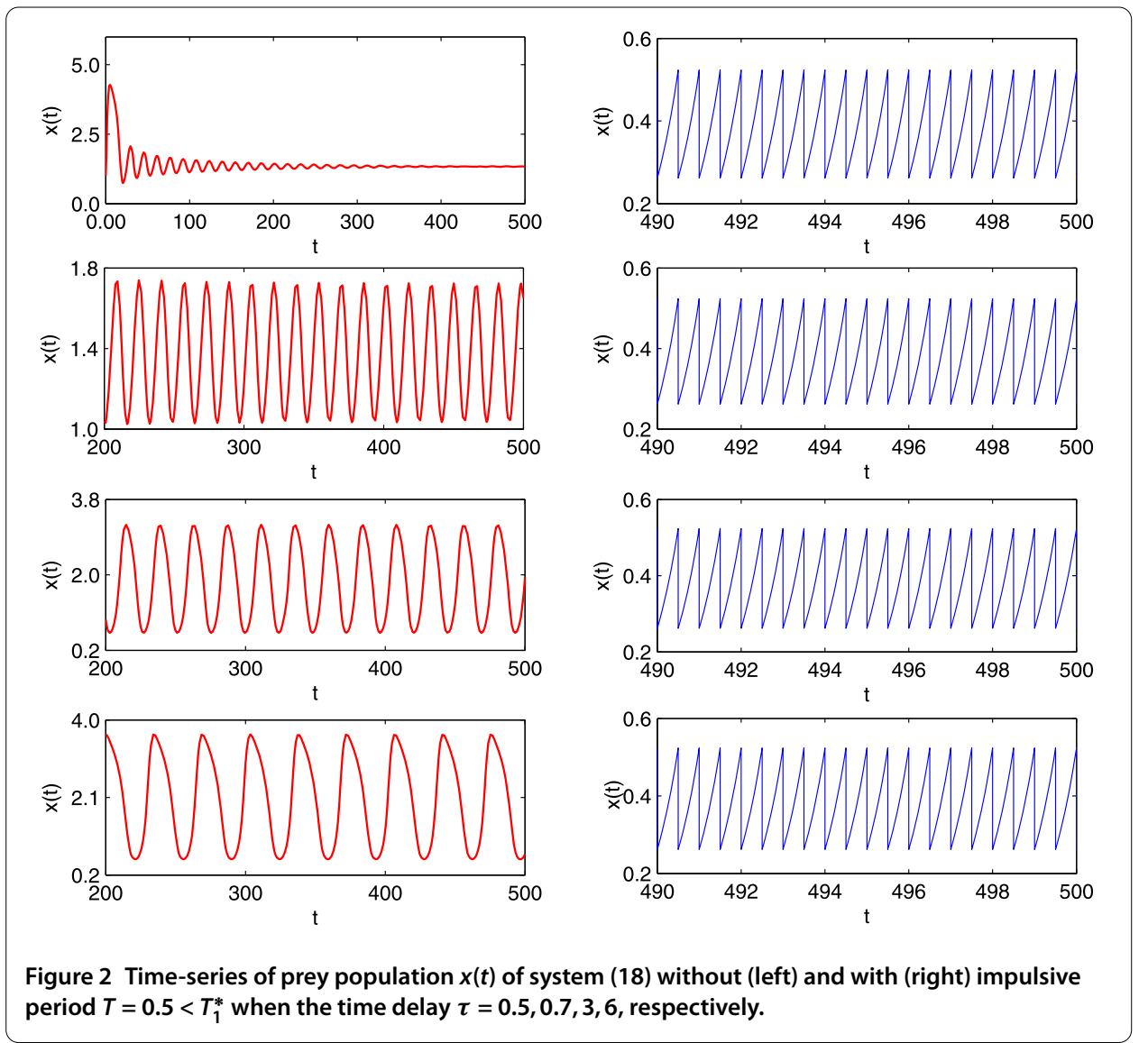

the following comparison system:

$$
\begin{cases}z_{3}^{\prime}(t)=r_{3} z_{3}(t)\left(1-\frac{z_{3}(t)}{K_{3}}\right), & t \neq n T, \\ \Delta z_{3}(t)=-p z_{3}(t), & t=n T,\end{cases}
$$

with initial condition $z_{3}\left(0^{+}\right)=x\left(0^{+}\right)$. Similarly, we can choose a $\varepsilon>0$ small enough such that

$$
x(t)>z_{3}^{*}(t)-\varepsilon \geq \frac{K_{3}\left[(1-p) e^{r_{3} T}-1\right]}{e^{r_{3} T}-1}-\varepsilon \triangleq m_{1}
$$

holds for sufficiently large $t$.

Remark 1 Let $(1-p) e^{r T}>1$ and

$$
F_{1}\left(f_{1}(p, T)\right)=\frac{k \alpha f_{1}^{2}(p, T)}{d\left(1+f_{1}^{2}(p, T)\right)}-1, \quad f_{1}(p, T)=\frac{K\left[(1-p) e^{r T}-1\right]}{(1-p)\left(e^{r T}-1\right)} .
$$

Note that $F_{1}\left(f_{1}(p, T)\right)$ is a monotonically increasing function with respect to $f_{1}(p, T)$, and $f_{1}(p, T)$ is a monotonically increasing function with respect to $T(T>-\ln (1-p) / r)$ and a monotonically decreasing function with respect to $p(0<p<1-\exp (-r T))$, since

$$
f_{1 p}^{\prime}(p, T)=\frac{-K}{\left(e^{r T}-1\right)(1-p)^{2}}<0, \quad f_{1 T}^{\prime}(p, T)=\frac{r p K e^{r T}}{(1-p)\left(e^{r T}-1\right)^{2}}>0 .
$$



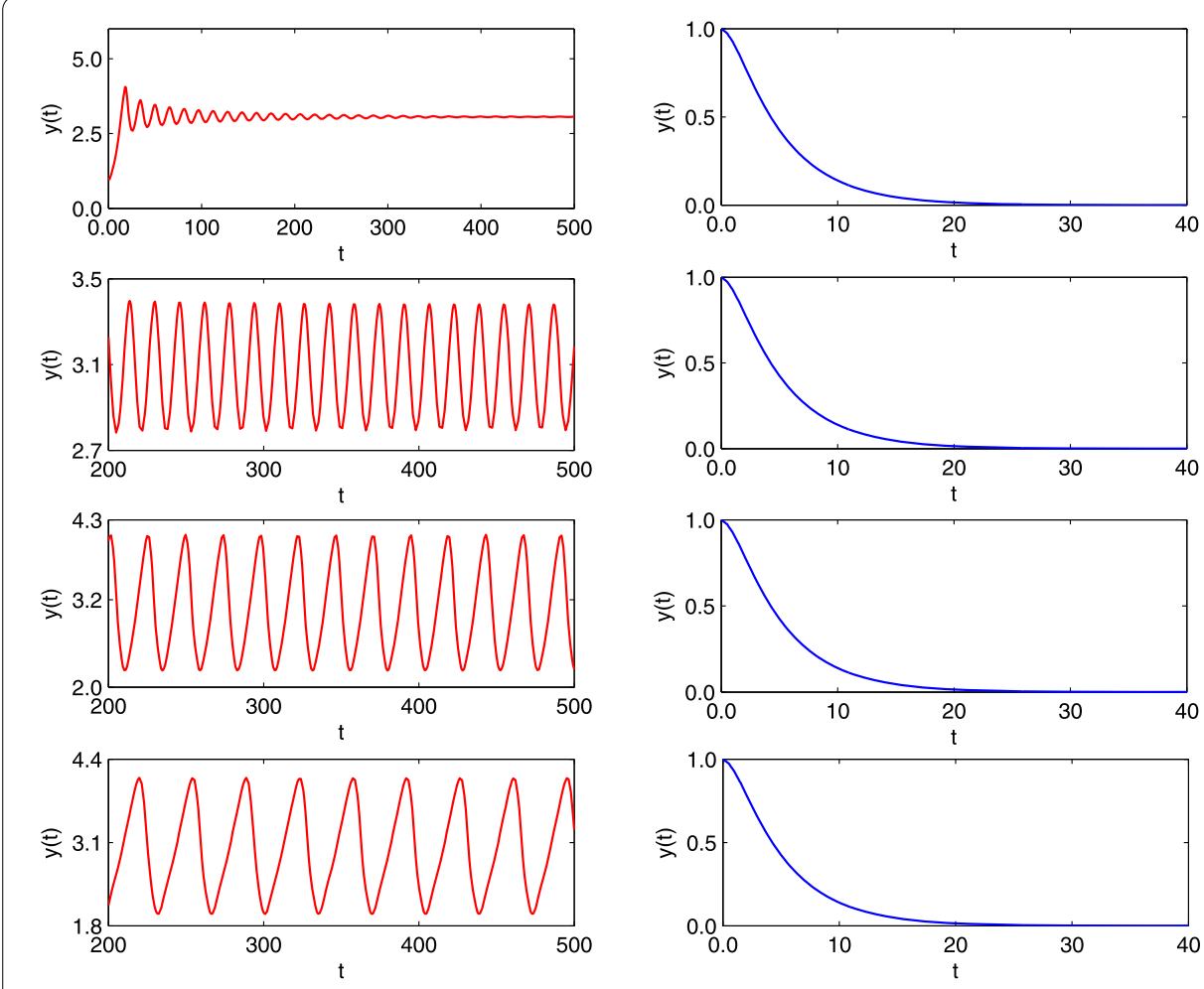

Figure 3 Time-series of predator population $y(t)$ of system (18) without (left) and with (right) impulsive period $T=0.5<T_{1}^{*}$ when time delay $\tau=0.5,0.7,3,6$, respectively.

So, there exists a unique $T_{1}^{*}$ such that $F_{1}\left(f_{1}\left(p, T_{1}^{*}\right)\right)=0$ if we fix $p$, similarly, there exists a unique $p_{1}^{*}$ such that $F_{1}\left(f_{1}\left(p_{1}^{*}, T\right)\right)=0$ if we fix $T$. Therefore, the condition $\Re_{1}<1$ is equivalent to $T<T_{1}^{*}\left(\right.$ or $\left.p>p_{1}^{*}\right)$, where $T_{1}^{*}\left(p_{1}^{*}\right)$ is the unique solution of $F_{1}\left(f_{1}(p, T)\right)=0$.

Similarly, let

$$
F_{2}\left(f_{2}(p, T)\right)=\frac{k \alpha f_{2}^{2}(p, T)}{d\left(1+f_{2}^{2}(p, T)\right)}-1, \quad f_{2}(p, T)=\frac{K\left[(1-p) e^{r T}-1\right]}{e^{r T}-1} .
$$

Note that $F_{2}\left(f_{2}(p, T)\right)$ is a monotonically increasing function with respect to $f_{2}(p, T)$, and $f_{2}(p, T)$ is a monotonically increasing function with respect to $T(T>-\ln (1-p) / r)$ and a monotonically decreasing function with respect to $p(0<p<1-\exp (-r T))$, since

$$
f_{2 p}^{\prime}(p, T)=-K e^{r T}<0, \quad f_{2 T}^{\prime}(p, T)=\frac{r p K e^{r T}}{\left(e^{r T}-1\right)^{2}}>0 .
$$

So, there exists a unique $T_{2}^{*}$ such that $F_{2}\left(f_{2}\left(p, T_{2}^{*}\right)\right)=0$ if we fix $p$, similarly, there exists a unique $p_{2}^{*}$ such that $F_{2}\left(f_{2}\left(p_{2}^{*}, T\right)\right)=0$ if we fix $T$. Therefore, the condition $\Re_{2}>1$ is equivalent to $T>T_{2}^{*}\left(\right.$ or $\left.p<p_{2}^{*}\right)$, where $T_{2}^{*}\left(p_{2}^{*}\right)$ is the unique solution of $F_{2}\left(f_{2}(p, T)\right)=0$.

Remark 2 Note that $f_{1}(p, T)>f_{2}(p, T)$, so $T_{1}^{*}<T_{2}^{*}$ for $F_{1}\left(f_{1}\left(p, T_{1}^{*}\right)\right)=0$ and $F_{2}\left(f_{2}\left(p, T_{2}^{*}\right)\right)=0$ with respect to the same value of the parameter $p$. Therefore, if $T<T_{1}^{*}$ the predatorextinction periodic solution is globally attractive and if $T>T_{2}^{*}$ the system has permanence. If system (1) is without time delay, according to [14], we know that there would be 


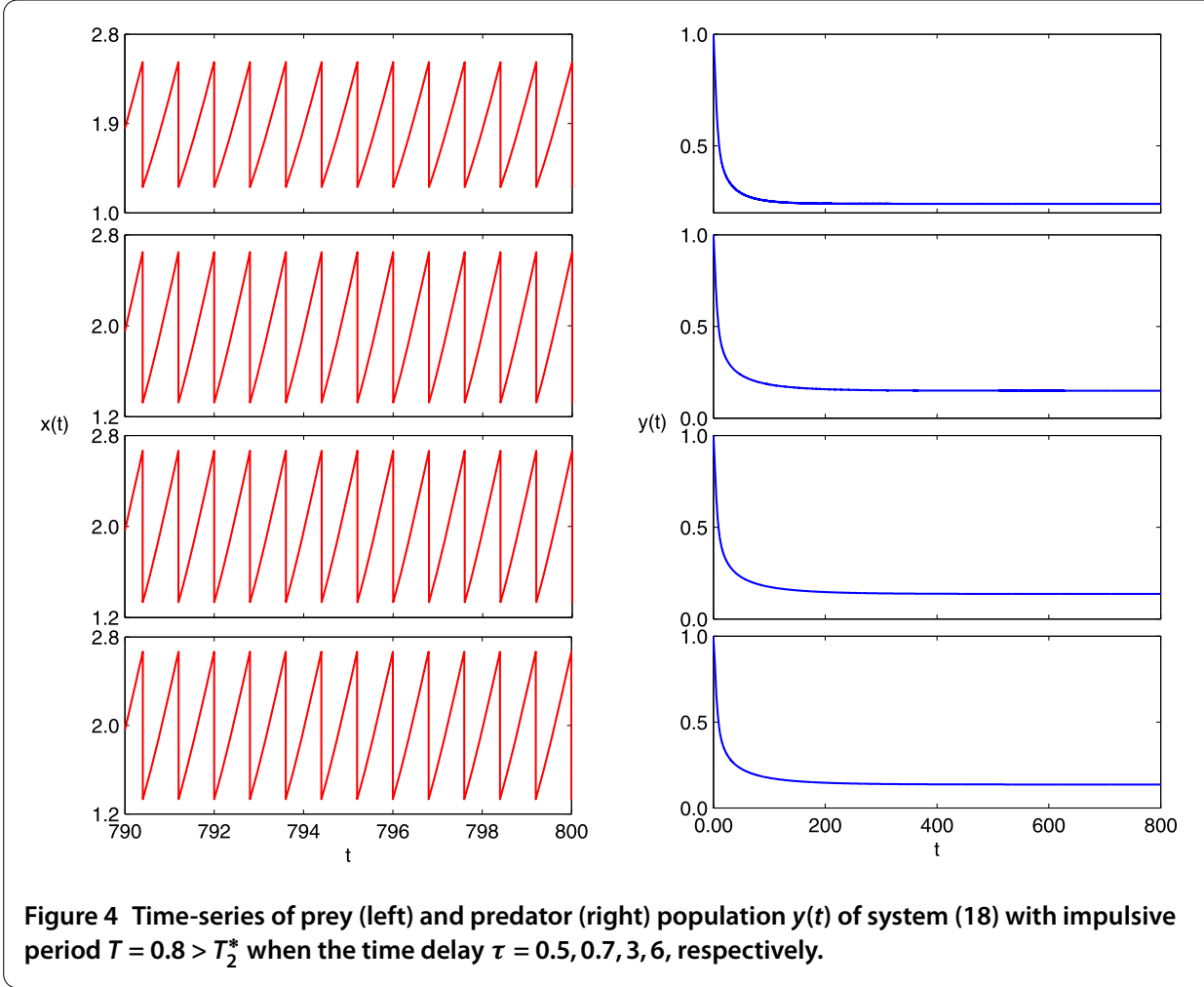

a threshold $T_{\max }$. If $T<T_{\max }$, then the prey-(or predator)-eradication periodic solution is locally asymptotically stable; if $T>T_{\max }$ the system is permanent. But we get two thresholds $T_{1}^{*}$ and $T_{2}^{*}$, and there is no information as regards the system when $T_{1}^{*}<T<T_{2}^{*}$. This is essentially different when system (1) is with or without time delay.

\section{Numerical analysis}

Numerical experiments are carried out to integrate the system by using the DDE23 algorithm method in MATLAB.

\subsection{Example 1}

We consider the following delayed Holling type-III response prey-predator system with impulsive perturbation:

$$
\left\{\begin{array}{r}
x^{\prime}(t)=1.5 x(t)\left(1-\frac{x(t)}{5}\right)-\frac{0.75 x^{2}(t) y(t)}{1+x^{2}(t)}, \\
y^{\prime}(t)=\frac{3 x^{2}(t-\tau) y(t-\tau)}{8\left[1+x^{2}(t-\tau)\right]}-0.24 y(t),
\end{array}\right\} \quad t \neq n T,
$$

where $r=1.5, K=5, \alpha=0.75, \beta=1, k=0.5, d=0.24$, with initial conditions $\left(\phi_{1}(t), \phi_{2}(t)\right)=$ $(1,1), t \in[-\tau, 0]$. From Remark 1 , we can get thresholds $T_{1}^{*}$ and $T_{2}^{*}$ when varying the values of parameter $p$ from 0.01 to 0.9 . From Figure 1 , we see that $T_{1}^{*}$ gets more and more close to $T_{2}^{*}$ when $p$ gets more and more close to zero.

According to [4, 6], there would be a time delay critical value $\tau_{0}$, and there is a Hopf bifurcation when the time delay $\tau$ crosses the critical value $\tau_{0}$ when system (18) is without 

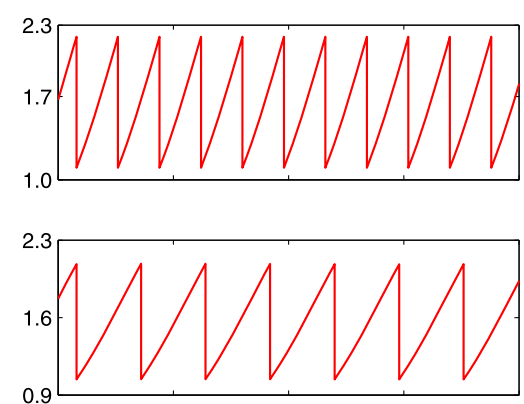

$x(t)$
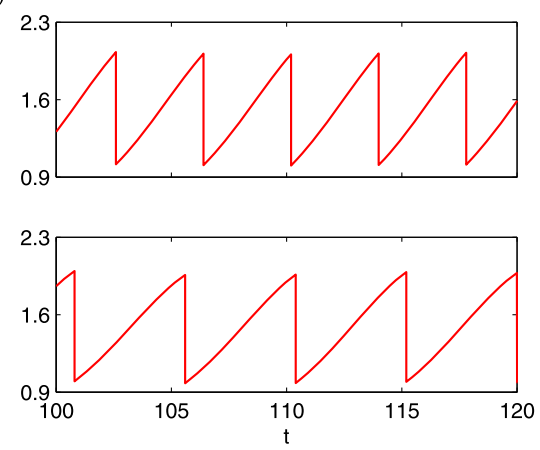
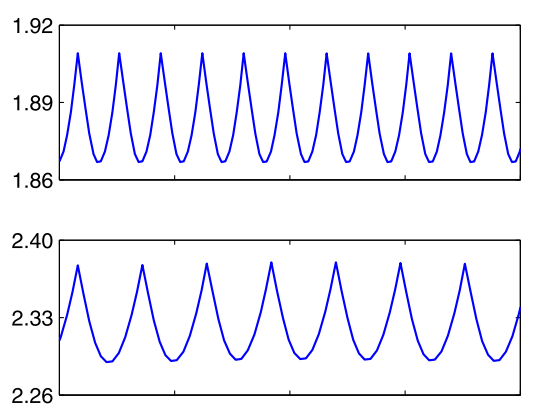

$y(t)$
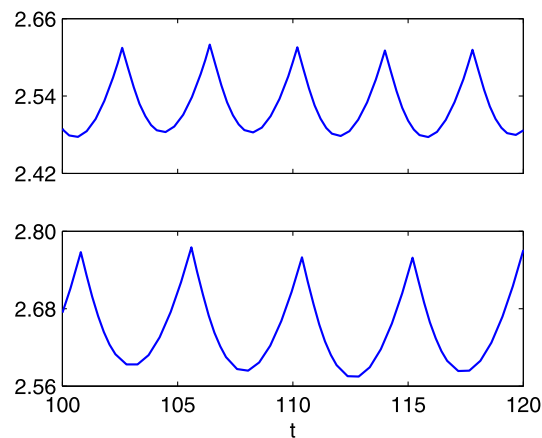

Figure 5 Time-series of prey (left) and predator (right) population $y(t)$ of system (18) with time delay $\tau=0.5$ when the impulsive period $T=1.8,2.8,3.8,4.8$, respectively.

impulsive perturbation (i.e. $p=0$ ). From the time-series of the prey population (Figure 2 (left)) and the predator population (Figure 3 (left)) with time delay $\tau=0.5,0.7,3,6$, respectively, we know that the time delay critical value $\tau_{0}$ is between 0.5 and 0.7 . The prey and predator populations are locally stable when the time delay $\tau \leq 0.5$ and coexist with sable cycles when $\tau \geq 0.7$.

From Theorem 3.1, Theorem 3.3, and Remark 1, one knows that if $T<T_{1}^{*}$ the predatorextinction periodic solution is globally attractive and if $T>T_{2}^{*}$ the system has permanence. From Figure 1 , we see $T_{1}^{*} \approx 0.5734$ and $T_{2}^{*} \approx 0.7634$ when $p=0.5$. Then, the predatorextinction periodic solution is globally attractive when $T=0.5<T_{1}^{*}$ (see Figure 2 (right) and Figure 3 (right)) where the time delay $\tau=0.5,0.7,3,6$, respectively. The system is permanent when $T=0.8>T_{2}^{*}$ where the time delay $\tau=0.5,0.7,3,6$, respectively (see Figure 4). Furthermore, when the time delay $\tau=0.5<\tau_{0}$ the predator and prey populations coexist with sable cycles, where the impulsive period $T=1.8,2.8,3.8,4.8$, respectively (see Figure 5). That is to say, an impulsive effect would destabilize the system under some conditions. If we increasing the time delay from 2 to 4.8 , that large time delay could stabilize the system (see Figure 6 and Figure 7).

\subsection{Example 2}

We consider the following delayed Holling type-III response predator-prey system with impulsive perturbation: 


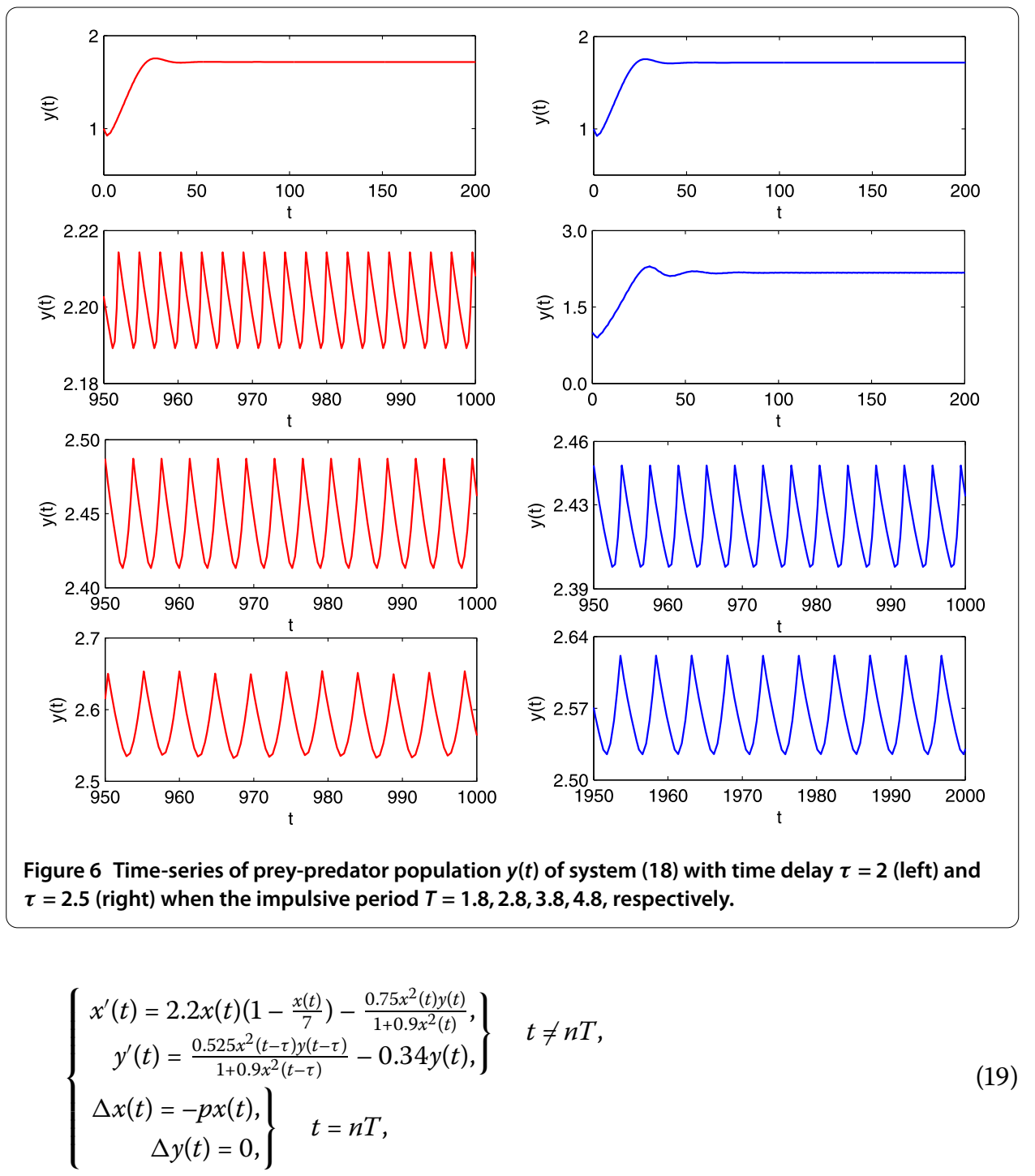

where $r=2.2, K=7, \alpha=0.75, \beta=0.9, k=0.7, d=0.34$, with initial condition $\left(\phi_{1}(s)\right.$, $\left.\phi_{2}(s)\right)=(1,1), s \in[-\tau, 0]$. First of all, we let $p=0.3, \tau=3$, and we consider the effect of the impulsive period $T$ on system (19). The bifurcation diagrams of the impulsive period $T$ over $[0.5,8.5]$ and $[6.8,8.5]$, show that system (19) has complex dynamics (see Figure 8 and Figure 9), including high-order periodic and quasi-periodic oscillating, period-doubling and period-halving bifurcations, and chaos and attractor crises. For example, there exist $5 T, 4 T, 3 T$ periodic solutions when $T=3.5,4.5,6.5$, respectively (see Figure 10). When $T$ increases from 6.8 to 7.1 , there is an attractor crisis leading to a chaotic solution (see Figure 11). These results imply that an impulsive effect could destroy the stability of the system and lead to multiple attractors, bifurcations, even chaos oscillations, which makes the dynamical behaviors very complex.

Second, we let $p=0.3, T=7$ and consider the effect of the time delay $\tau$ on system (19). The bifurcation diagrams of time delay $\tau$ over $[0.2,7.0]$ and $[2.5,3.1]$, show that system (19) has very complex dynamic behaviors (see Figure 12 and Figure 13). These results imply that the time delay would make the dynamical behaviors more complex. For example, there is a cascade of period-doubling bifurcations leading to chaos (see Figure 14). 

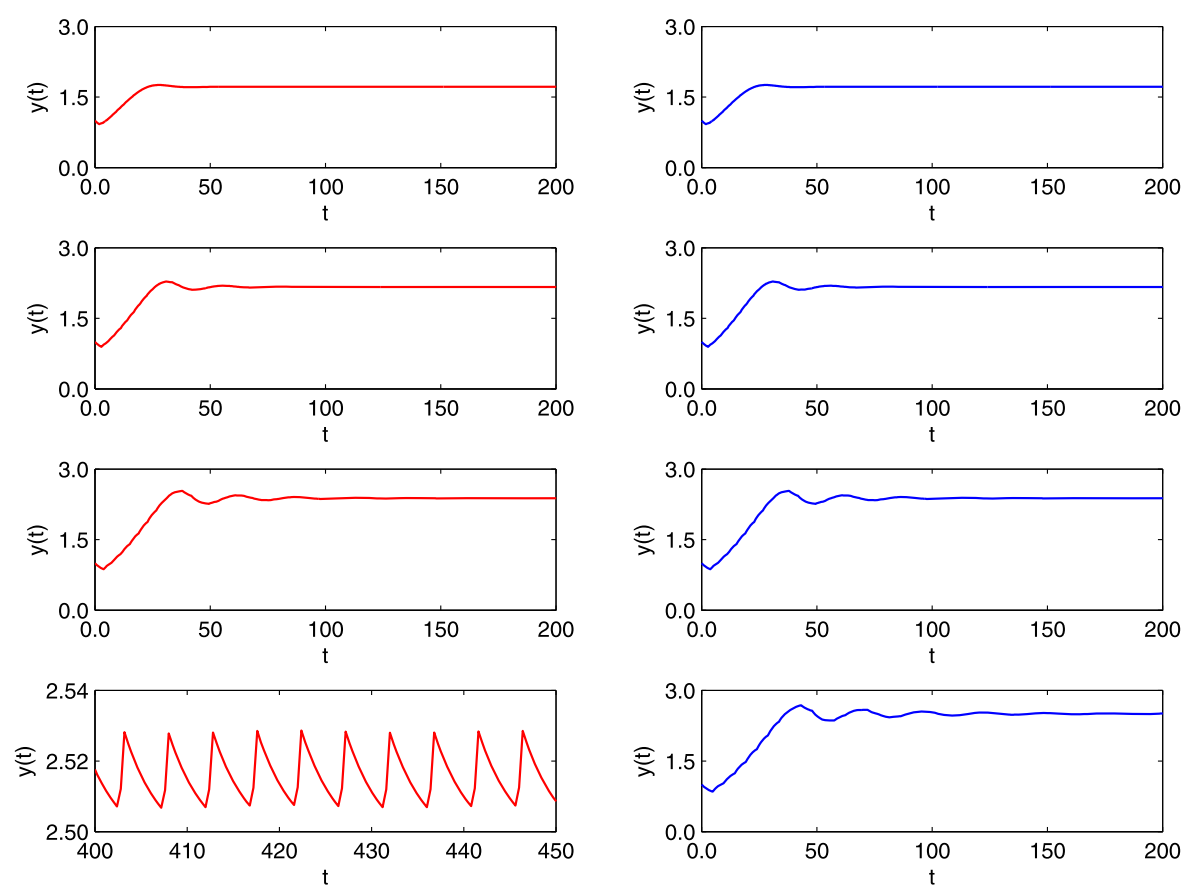

Figure 7 Time-series of prey predator population $y(t)$ of system (18) with time delay $\tau=4$ (left) and $\tau=4.8$ (right) when the impulsive period $T=1.8,2.8,3.8,4.8$, respectively.
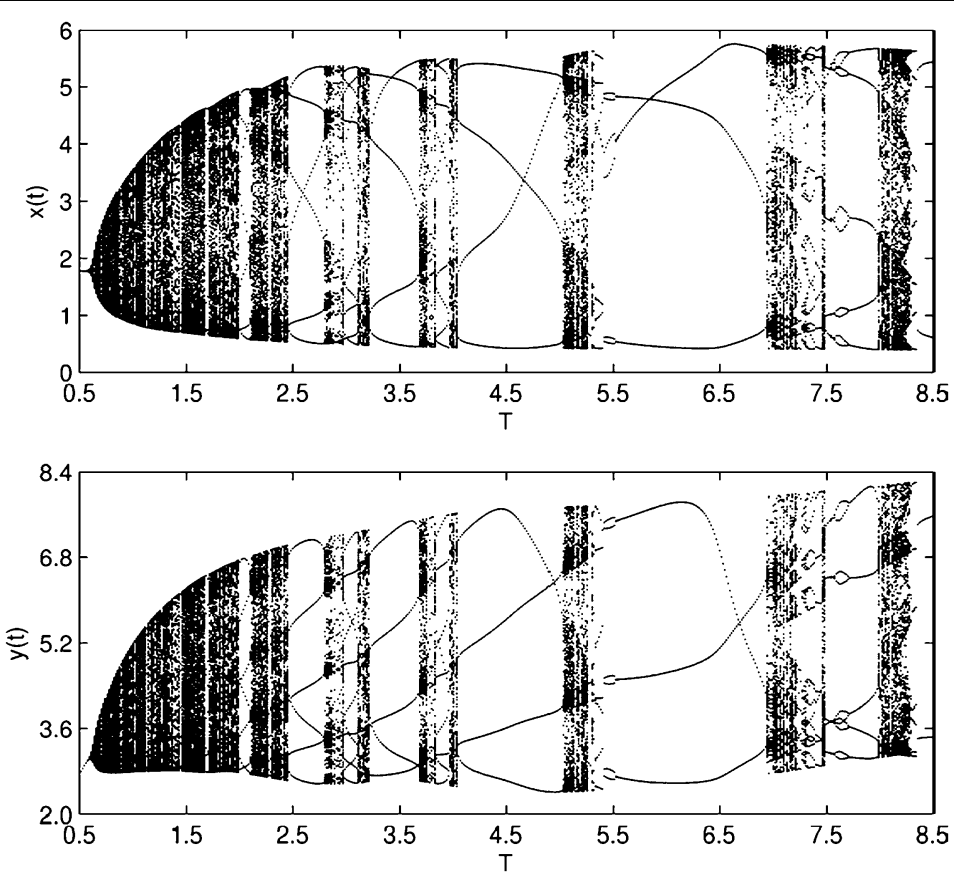

Figure 8 Bifurcation diagrams of system (19): prey population $x(t)$ (top) and predator population $y(t)$ (bottom) when $p=0.3, \tau=3$ with impulsive period $T$ over $[0.5,8.5]$. 

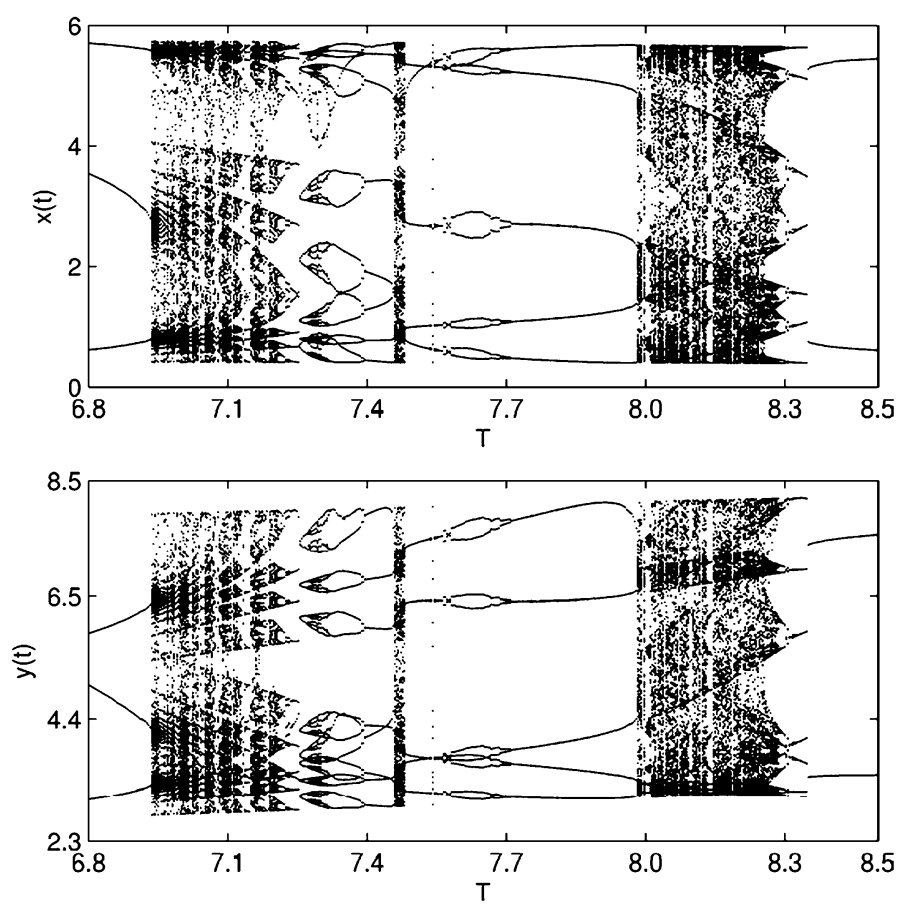

Figure 9 Bifurcation diagrams of system (19): prey population $x(t)$ (top) and predator population $y(t)$ (bottom) when $p=0.3, \tau=3$ with impulsive period $T$ over $[6.9,7.5]$.
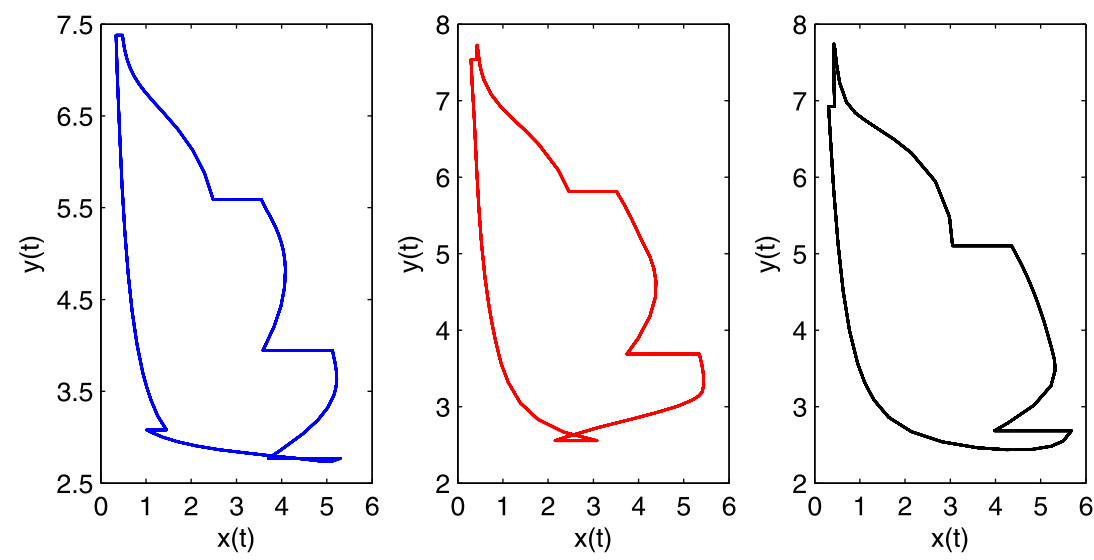

Figure 10 Phase portrait of the system (19). 5T, 4T, $3 T$ periodic solution when $T=3.5,4.5,6.5$, respectively.

Finally, we let $\tau=7, T=7$, and we investigate the impulsive perturbation proportionality constant $p$ on system (19). The bifurcation diagrams of impulsive perturbation proportionality constant $p$ over $[0.2,0.74]$ show that system (19) has complex dynamics (see Figure 15). These results imply that the impulsive perturbation proportionality constant $p$ would make the dynamical behaviors more complex, too. From the bifurcation diagrams (Figures 8, 9, 12, 13 and 15), we know that the parameters impulsive period $T$, time delay $\tau$, and impulsive perturbation proportionality constant $p$ would be important factors to affect the dynamic behaviors of the system, and make the system subject to complex dynamical behaviors. 

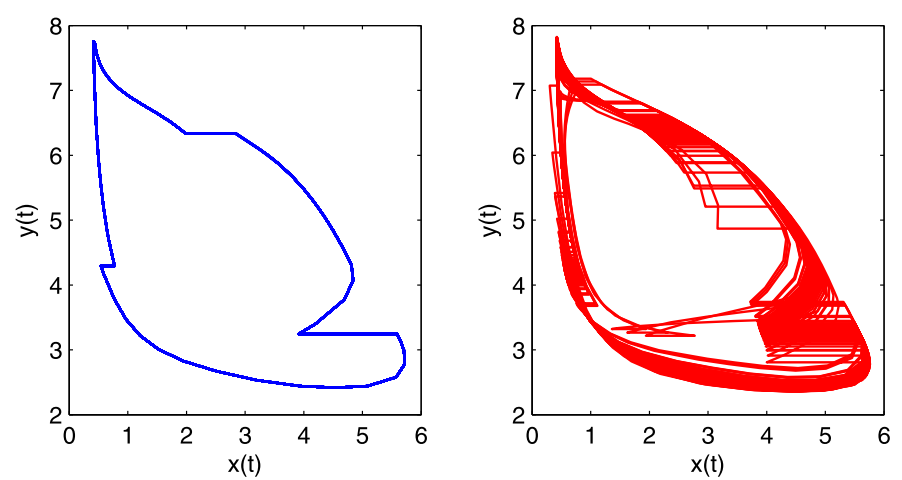

Figure 11 Crisis are shown: from left to right there is a crisis that the chaos suddenly appears when $T=6.93,6.94$, respectively.
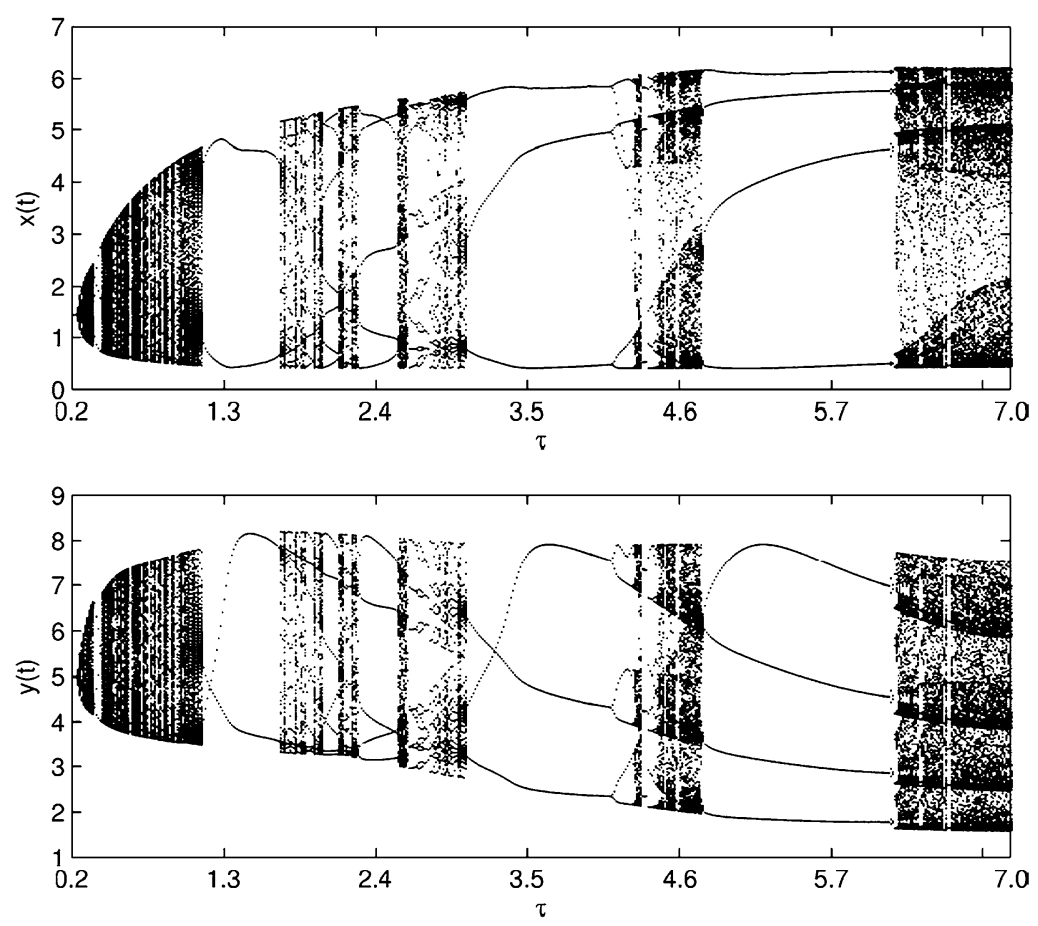

Figure 12 Bifurcation diagrams of system (19): prey population $x(t)$ (top) and predator population $y(t)$ (bottom) when $p=0.3, T=7$ with time delay $\tau$ over $[0.2,7.0]$.

\section{Conclusion}

In this paper, we have investigated a prey-predator system with constant gestation time delay and impulsive perturbation on the prey in detail. We have shown that there exists a globally attractive predator-extinction periodic solution when the impulsive period $T$ is less than the critical value $T_{1}^{*}$. The system is permanent when the impulsive period $T$ is larger than the critical value $T_{2}^{*}$. Therefore, we get two thresholds $T_{1}^{*}$ and $T_{2}^{*}$, and there is no information as regards the system (1) when $T_{1}^{*}<T<T_{2}^{*}$. If the system (1) is without time delay, then $T_{1}^{*}=T_{\max }=T_{2}^{*}$ would be the unique threshold. This is essentially different when system (1) is with or without time delay. 

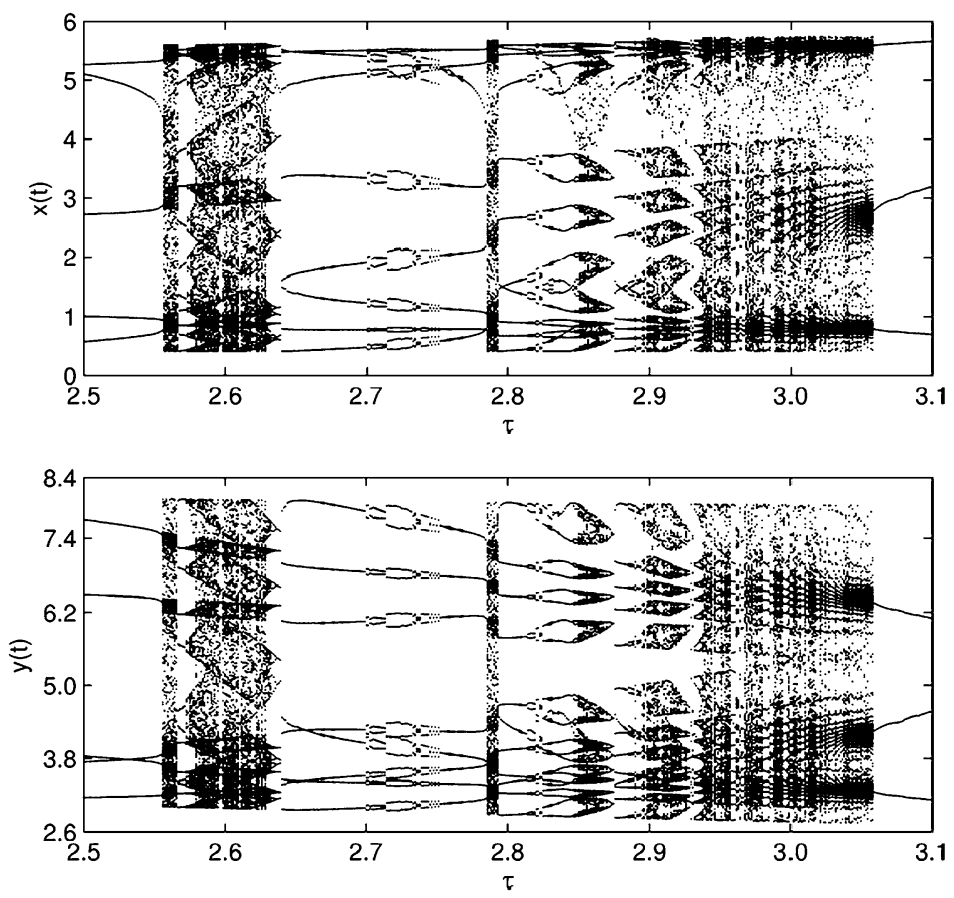

Figure 13 Bifurcation diagrams of system (19): prey population $x(t)$ (top) and predator population $y(t)$ (bottom) when $p=0.3, T=7$ with time delay $\tau$ over [2.5,3.1].
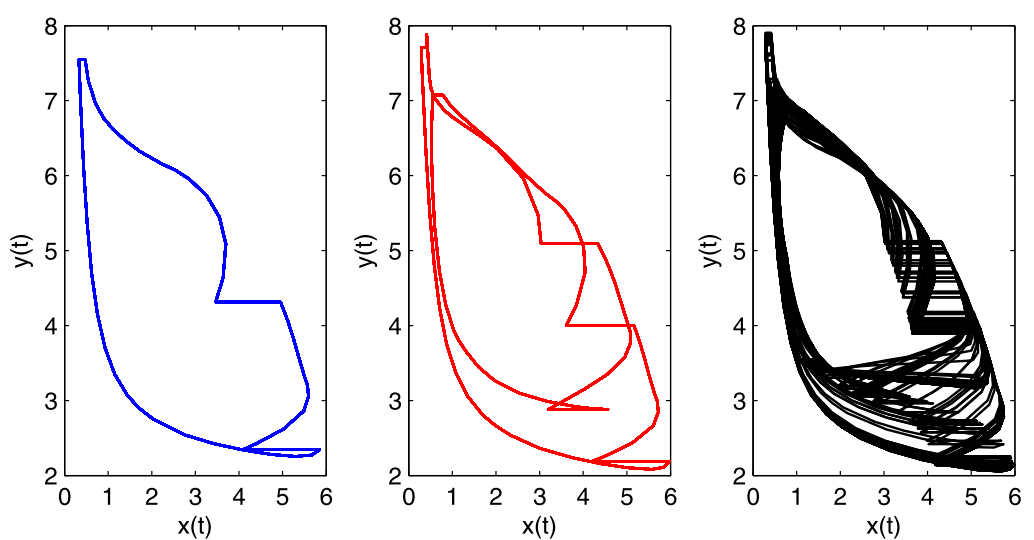

Figure 14 Period-doubling bifurcation leads to chaos. From left to right: phase portrait of $T$-periodic, $2 T$-periodic solutions and chaos when $\tau=4.1,4.2,4.3$, respectively.

Numerical examples show that system (1) have various kinds of periodic oscillations, including high-order periodic and quasi-periodic oscillations, chaotic oscillations. These results imply that the parameters of impulsive period $T$, time delay $\tau$, and impulsive perturbation proportionality constant $p$ would be important factors to affect the dynamic behaviors of the system (1), and make the system (1) subject to complex dynamical behaviors. That large time delay could stabilize the system, an impulsive effect could destabilize the system. Therefore, the dynamical behaviors would be more complex when the system is subject to both time delay and an impulsive effect. 

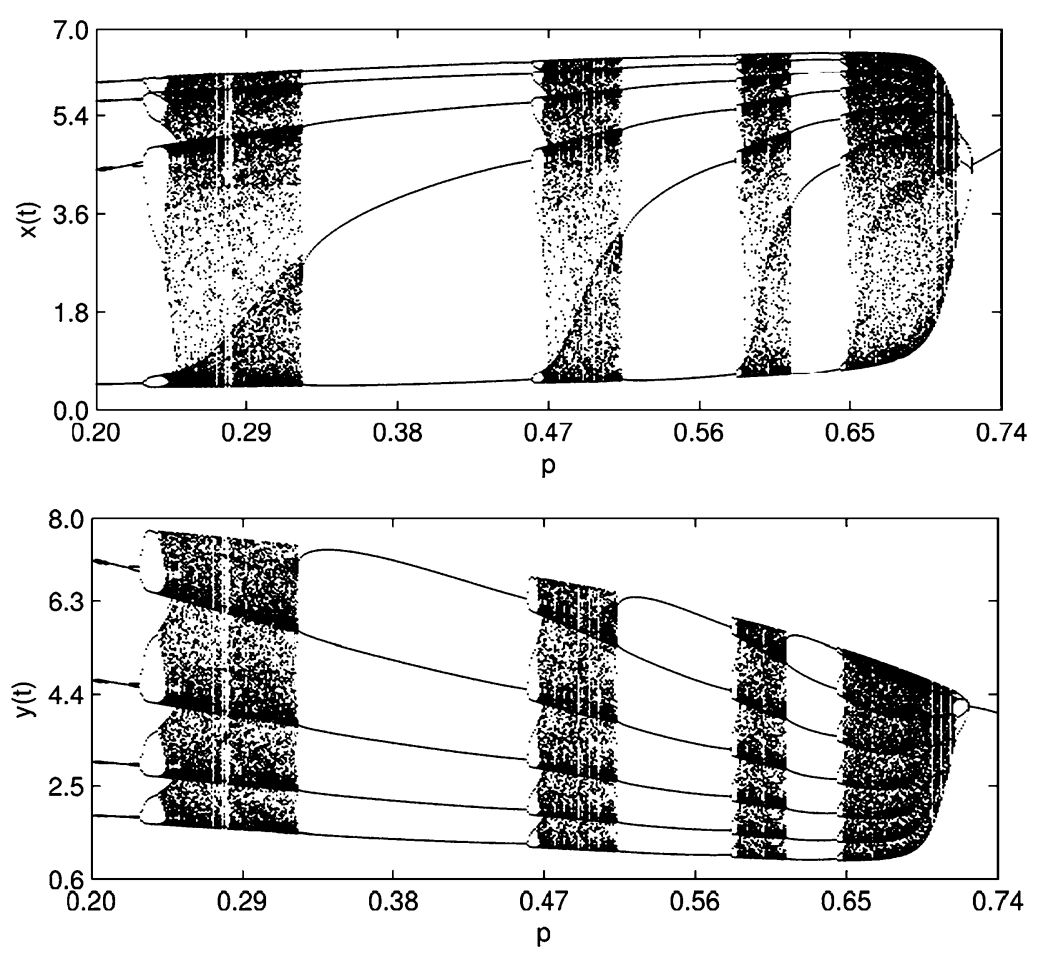

Figure 15 Bifurcation diagrams of system (19): prey population $x(t)$ (top) and predator population $y(t)$ (bottom) when $\tau=7, T=7$ with parameter $p$ over [0.2, 0.74].

\section{Competing interests}

The authors declare that they have no competing interests.

\section{Authors' contributions}

SL carried out the main part of this manuscript. WL participated in the discussion and gave the examples. All authors read and approved the final manuscript.

\section{Acknowledgements}

This work was supported by the Joint Natural Science Foundation of Guizhou Province (Nos. LKQS[2013]12 and LH[2014]7437); Foundation of Qiannan Normal College for Nationalities (Nos. 2014ZCSX03 and 2014ZCSX11).

Received: 19 October 2015 Accepted: 21 January 2016 Published online: 05 February 2016

\section{References}

1. Li, S, Xue, Y, Liu, W: Hopf bifurcation and global periodic solutions for a three-stage-structured prey-predator system with delays. Int. J. Inf. Syst. Sci. 8(1), 142-156 (2012)

2. Li, S, Liu, W, Xue, X: Bifurcation analysis of a stage-structured prey-predator system with discrete and continuous delays. Appl. Math. 4(7), 1059-1064 (2013)

3. Li, S, Xiong, Z: Bifurcation analysis of a predator-prey system with sex-structure and sexual favoritism. Adv. Differ. Equ. 2013, Article ID 219 (2013)

4. Wang, L, Xu, R, Feng, G: A stage-structured predator-prey system with time delay and Holling type-III functional response. Int. J. Pure Appl. Math. 48(2), 53-66 (2008)

5. Zou, W, Xie, J, Xiong, Z: Stability and Hopf bifurcation for an eco-epidemiology model with Holling-III functional response and delays. Int. J. Biomath. 1(3), 377-389 (2008)

6. Zhang, $X, X u, R, G a n, Q$ : Periodic solution in a delayed predator prey model with Holling type III functional response and harvesting term. World J. Model. Simul. 7(1), 70-80 (2011)

7. Das, U, Kar, TK: Bifurcation analysis of a delayed predator-prey model with Holling type III functional response and predator harvesting. J. Nonlinear Dyn. 2014, Article ID 543041 (2014)

8. Zhang, Z, Yang, H, Fu, M: Hopf bifurcation in a predator-prey system with Holling type III functional response and time delays. J. Appl. Math. Comput. 44(1-2), 337-356 (2014)

9. Fan, Y, Wang, L: Multiplicity of periodic solutions for a delayed ratio-dependent predator-prey model with Holling type III functional response and harvesting terms. J. Math. Anal. Appl. 365(2), 525-540 (2010)

10. Cai, Z, Huang, L, Chen, H: Positive periodic solution for a multi species competition-predator system with Holling III functional response and time delays. Appl. Math. Comput. 217(10), 4866-4878 (2011)

11. Li, G, Yan, J: Positive periodic solutions for neutral delay ratio-dependent predator-prey model with Holling type III functional response. Appl. Math. Comput. 218(8), 4341-4348 (2011) 
12. $\mathrm{Xu}, \mathrm{C}, \mathrm{Wu}, \mathrm{Y}, \mathrm{Lu}, \mathrm{L}:$ Permanence and global attractivity in a discrete Lotka-Volterra predator-prey model with delays. Adv. Differ. Equ. 2014, Article ID 208 (2014)

13. Li, S: Complex dynamical behaviors in a predator-prey system with generalized group defense and impulsive control strategy. Discrete Dyn. Nat. Soc. 2013, Article ID 358930 (2013)

14. Li, S, Xiong, Z, Wang, X: The study of a predator-prey system with group defense and impulsive control strategy. Appl. Math. Model. 34(9), 2546-2561 (2010)

15. Su, H, Dai, B, Chen, Y, et al.: Dynamic complexities of a predator-prey model with generalized Holling type III functional response and impulsive effects. Comput. Math. Appl. 56(7), 1715-1725 (2008)

16. Fan, X, Jiang, F, Zhang, H: Dynamics of multi-species competition-predator system with impulsive perturbations and Holling type III functional responses. Nonlinear Anal., Theory Methods Appl. 74(10), 3363-3378 (2011)

17. Liu, Z, Zhong, S: An impulsive periodic predator-prey system with Holling type III functional response and diffusion. Appl. Math. Model. 36(12), 5976-5990 (2012)

18. Yan, C, Dong, L, Liu, M: The dynamical behaviors of a nonautonomous Holling III predator-prey system with impulses. J. Appl. Math. Comput. 47(1), 193-209 (2015)

19. Tan, R, Liu, W, Wang, Q: Uniformly asymptotic stability of almost periodic solutions for a competitive system with impulsive perturbations. Adv. Differ. Equ. 2014, Article ID 2 (2014)

20. $\mathrm{Xu}, \mathrm{L}, \mathrm{Wu}, \mathrm{W}$ : Dynamics of a nonautonomous Lotka-Volterra predator-prey dispersal system with impulsive effects. Adv. Differ. Equ. 2014, Article ID 264 (2014)

21. Ju, Z, Shao, Y, Kong, W: An impulsive prey-predator system with stagestructure and Holling II functional response. Adv. Differ. Equ. 2014, Article ID 280 (2014)

22. Jia, J, Cao, H: Dynamic complexities of Holling type II functional response predator-prey system with digest delay and impulsive. Int. J. Biomath. 2(2), 229-242 (2009)

23. Bainov, DD, Simeonov, DD: Impulsive Differential Equations: Periodic Solutions and Applications. Longman, Harlow (1993)

24. Lakshmikantham, V, Bainov, DD, Simeonov, PS: Theory of Impulsive Differential Equations. World Scientific, Singapore (1989)

25. Kuang, Y: Delay Differential Equations with Applications in Population Dynamics. Academic Press, New York (1993)

\section{Submit your manuscript to a SpringerOpen ${ }^{\ominus}$ journal and benefit from:}

- Convenient online submission

Rigorous peer review

- Immediate publication on acceptance

- Open access: articles freely available online

- High visibility within the field

- Retaining the copyright to your article 